\title{
A NEW OPTIMALITY BASED REPETITIVE CONTROL ALGORITHM FOR DISCRETE-TIME SYSTEMS
}

\author{
J. Hätönen ${ }^{*, \dagger}$, D. H. Owens ${ }^{\dagger}$, R. Ylinen * \\ * Systems Engineering Laboratory, University of Oulu, P.O.BOX 4300, FIN-90014 University of Oulu, Finland \\ $\dagger$ Department of Automatic Control and Systems Engineering, University of Sheffield, Mappin Street, Sheffield S1 3JD, UK
}

Keywords: Repetitive Control, Optimal Control, Polynomial Systems Theory.

\begin{abstract}
In this paper it is shown that discretisation of a class of wellknown continuous-time repetitive control algorithms will destroy stability. In order to overcome this problem, a new optimality based Repetitive Control algorithm is proposed for discrete-time systems. Under mild technical conditions on the plant the algorithm will result in asymptotic convergence for an arbitrary $T$-periodic reference signal and an arbitrary discretetime linear time-invariant plant. Simulations highlight the different theoretical findings in this paper. Copyright (C) 2003 IFAC
\end{abstract}

\section{Introduction}

Many signals in engineering are periodic, or at least they can be accurately approximated by a periodic signal over a large time interval. This is true, for example, of most signals associated with engines, electrical motors and generators, converters, or machines performing a task over and over again. Hence it is an important control problem to try to track a periodic signal with the output of the plant or try to reject a periodic disturbance acting on a control system.

In order to solve this problem, a relatively new research area called Repetitive Control has emerged in the control community. The idea is to use information from previous periods to modify the control signal so that the overall system would 'learn' to track perfectly a given $T$-periodic reference signal. The first paper that uses this ideology seems to be (Inouye et al., 1981), where the authors use repetitive control to obtain a desired proton acceleration pattern in a proton synchotron magnetic power supply.

Since then repetitive control has found its way to several practical applications, including robotics (Kaneko and Horowitz, 1997), motors (Kobayashi et al., 1999), rolling processes (Garimella and Srinivasan, 1996) and rotating mechanisms (Fung et al., 2000). However, most of the existing Repetitive Control algorithms are designed in continuous time, and they either don't give perfect tracking or they require that original process is positive real. In order to overcome these limitations, in this paper a new optimality based Repetitive Control algorithm is introduced for linear time-invariant discrete-time systems, which will result in perfect tracking under mild technical assumptions.

\section{Problem definition and earlier work}

As a starting point in continuous-time Repetitive Control (RC) it is assumed that a mathematical model

$$
\begin{aligned}
& \dot{x}(t)=A x(t)+B u(t) \\
& y(t)=C x(t)+D u(t)
\end{aligned}
$$

of the plant in question exists with $x(0)=x_{0}, t \in[0, \infty)$. Furthermore, $A, B, C$ and $D$ are finite-dimensional matrices of appropriate dimensions. From now on it is assumed that $D=0$, because in practise it very rare to find a system where the input function $u(t)$ has an immediate effect on the output variable $y(t)$. Furthermore, a reference signal $r(t)$ is given, and it is known that $r(t)=r(t+T)$ for a given $T$ (in other words the actual shape of $r(t)$ is not necessarily known). The control design objective is to find a feedback controller that makes the system (1) to track the reference signal as accurately as possible (i.e. $\lim _{t \rightarrow \infty} e(t)=0, e(t):=r(t)-y(t)$ ), under the assumption that the reference signal $r(t)$ is $T$-periodic. As was shown by (Francis and Wonhan, 1975), a necessary condition for asymptotic convergence is that a controller

$$
[M u] t=[N e](t)
$$

where $M$ and $N$ are suitable operators, has to have an internal model or the reference signal inside the operator $M$. Because $r(t)$ is $T$-periodic, its internal model is $1-\sigma_{T}$, where $\left[\sigma_{T} v\right](t)=v(t-T)$ for $v: \mathbb{R} \rightarrow \mathbb{R}$. Hence in (Yamamoto, 1993) it was suggested, that one possible (and obviously computationally simple) RC algorithm for the SISO case could be

$$
u(t)=u(t-T)+e(t)
$$

This algorithm has been analysed by several authors, see for example (Yamamoto, 1993), (Arimoto and Naniwa, 2000) and (Owens et al., 2001). It turns out that if the system (1) is positive real (PR) then for $e(\cdot) \in L_{2}[0, \infty)$ (this does not imply that $\left.\lim _{t \rightarrow \infty} e(t)=0\right)$ ). The definition of a positive real system from (Anderson and Vongpanithred, 1973) is given in the following

Definition 1 (A PR system - continuous-time case) Consider the transfer function matrix $G(s)$ of the system (1) where $G(s)=C(s I-A)^{-1} B+D$. System (1) is positive real

1) Each element of the transfer function $G(s)$ are analytic for $\operatorname{Re}[s]>0$ 
2) $G(s)$ is real for real positive $s$

3) $G(s)+G(s)^{*} \geq 0$ for $\operatorname{Re}[s] \geq 0$

where the superscript ${ }^{*}$ denotes complex conjugation.

To see why positivity is required in the SISO case, consider now the following 'relaxed' algorithm

$$
u(t)=\alpha u(t-T)+K e(t)
$$

where $\alpha \in(0,1)$ is a relaxation parameter and $K \in \mathbb{R}, K>0$. An equivalent representation of the algorithm is given by

$$
[1+K G u](t)=\alpha u(t-T)+K r(t)
$$

where it is assumed that $r(\cdot) \in L_{2}^{l o c}[0, \infty)$. The Laplacetransform of (5) becomes (assuming that $G(s)$ is stable with zero initial conditions)

$$
u(s)=\frac{\alpha e^{-s T}}{1+K G(s)} u(s)+\frac{K r(s)}{1+K G(s)}
$$

An easy application of the small-gain theorem (see (Zhou et al., 1996)) gives that a sufficient condition for stability (i.e. $u(\cdot) \in$ $\left.L_{2}[0, \infty)\right)$ is that

$$
\sup _{\omega \geq 0}\left|\frac{\alpha}{1+K G(j \omega)}\right|<1
$$

where it is assumed that $\frac{\alpha}{1+K G(s)}$ is a stable system. Note that if $\alpha=1$, this inequality is never met if $G(s)$ is strictly proper, because for a strictly proper $G(s), \lim _{\omega \rightarrow \infty} G(j \omega)=0$ and therefore $\lim _{\omega \rightarrow \infty}\left|\frac{1}{1+K G(j \omega)}\right|=1$. Stability can achieved, however, if $\alpha$ is selected to be sufficiently small, resulting in a non-zero tracking error.

It can be shown that the sufficient condition (7) implies that the control law converges to a $T$-periodic solution. In the limit the control law (4) becomes

$$
u(t)=\frac{K}{1-\alpha} e(t)
$$

and $y(t)$ to converges to a $T$-periodic solution

$$
y(t)=\frac{\frac{K}{1-\alpha} G}{1+\frac{K}{1-\alpha} G} r(t)
$$

If $\alpha$ now tends to one (giving the original law in previous section), it is clear that this simple control law (5) generates an infinite feedback gain in the limit based on $(8)$ and $y(t)$ converges to $r(t)$ based on (9). However, a positive real system can tolerate with infinite feedback gain, as was shown for example in (Owens et al., 2001) with a Lyapunov-based approach for the MIMO multi-periodic case. Thefore (9) is stable for an arbitrary $\alpha \in[0,1]$, and $\alpha=1$ results in $y(t)=r(t)$.

In the single-periodic SISO case there is a more visual way to prove that positive realness is a sufficient condition for convergence with the algorithm (3): as a starting point Definition
1 can be understood as a statement that the Nyquist diagram of the positive real system $G$ lies in the right-half plane (see Fig. 1). Furthermore, it is a standard result in classical control theory that a control law $u(t)=-K e(t)$ results in a stable closed-loop system if the Nyquist diagram of $K G$ does not encircle the critical point $(-1,0)$. However, if $G$ lies in the righthalf plane, and $G$ is multiplied with a positive $K$, the resulting Nyquist diagram will be still in the right-half plane as shown in Fig. 1, and consequently a positive real system can tolerate an arbitrary large feedback gain $K$.

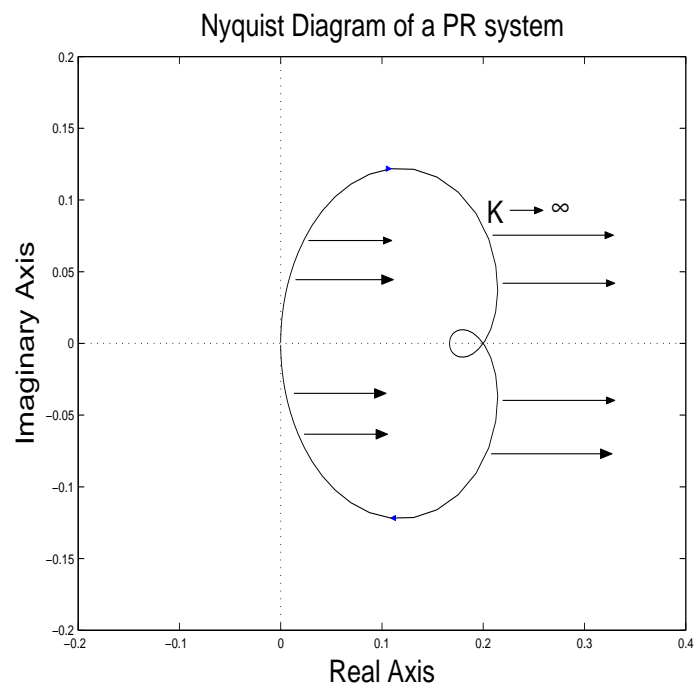

Figure 1: The Nyquist diagram of a positive real system

\section{A fundamental problem caused by implemen- tation}

As was shown in the previous section, the simple repetitive control law (3) will result in convergent learning when the original continuous-time system is positive real. However, this result is not as useful as it sounds, because in practise it is impossible to implement a delay block using analogue components. Hence it is natural to ask, that if the original plant is discretized with zero-order hold (which models exactly the behaviour of the continuous-time system at the sampled time points), does the sampled system remain positive real if the original continuous time plant is strictly proper and positive real? First the definition of a positive real system in the discrete-time case is needed (see (Desoer and Vidyasagar, 1975) for details):

Definition 2 (A positive real system - discrete-time case) Consider the following LTI discrete-time system

$$
\begin{aligned}
& x(t+1)=A x(t)+B u(t) \\
& y(t)=C x(t)+D u(t)
\end{aligned}
$$

and the corresponding transfer function matrix $G(z)=$ $C(z I-A)^{-1} B+D$. The system (10) is said to be positive-real, if 
1) $G(z)$ is analytic for $|z|>1$

2) $G(z)$ is real for real positive $z$

3) $\operatorname{Re}\left[G\left(e^{j \theta}\right)\right] \geq 0$ for all $\theta \in[0,2 \pi]$ (i.e. the Nyquist diagram of $G(z)$ lies in the right-half plane)

The next proposition shows a rather disappointing result that for a discrete-time system (10) a necessary condition for positive realness is that $D \neq 0$, and hence strictly proper discretetime systems cannot cope with infinite feedback gain:

Proposition 1 Suppose that the system (10) is positive real and $C B \neq 0$ (the assumption that $C B \neq 0$ is almost always true for a continuous-time system sampled with zero-order hold). This implies that $D \neq 0$.

Proof. Assume that $D=0$ but (10) is positive real. Consider now the transfer function of (10) given by

$$
G(z)=C(z I-A)^{-1} B
$$

It is a well-known result from classical control theory that the stability of the system can be analysed with the root-locus method, where the roots of the equation $1+K G(z)$ are plotted on the complex plane for different values of $K>0$ (see (Ogata, 1973)). Furthermore, according to (Ogata, 1973), the poles of the closed-loop system approach the open-loop zeros (zeros of $G(z)$ ) when $K \rightarrow \infty$. Because the relative degree of $G(z)$ is one, it has a zero at 'infinity'. Hence one of the closed-loop poles converges towards infinity as $K \rightarrow \infty$, and therefore it cannot stay inside the unit circle for arbitrary large values of $K$. Consequently the Nyquist-diagram of $G(z)$ does not lie entirely in the right-half plane, and hence system (10) is not positive-real when $D=0$.

However, a similar reasoning as in the previous section shows that if the algorithm $u(t)=u(t-T)+K e(t)$ (now as a discrete-time law) gives convergent learning, the algorithm results in infinite feedback gain. Therefore it is impossible to implement the control law (4) without instability but one has to resort to the 'relaxed' version (5) (where $\alpha$ is typically replaced with a more advanced causal filter), which does not give perfect tracking. To overcome this problem, in the next section a computationally more complex algorithm is proposed for the discrete-time case, which results in perfect tracking.

Remark 1 It is easy to show in the continuous-time case with $D=0$, that an infinitely small time delay will destroy stability. This is due to the fact that an equivalent condition for positiverealness is that the phase-lag of the plant $G(j \omega)$ for $\omega>0$ does not exceed $-90^{\circ}$. A time delay, however, causes phase-lag

$$
\angle e^{-j \omega T}=-\omega T \mathrm{rad}
$$

Furthermore, because $D=0, \angle G(j \omega) \rightarrow-\pi / 2 \mathrm{rad}$ as $\omega \rightarrow \infty$, and if a time delay is connected in series with $G(s)$, there exists $\omega_{0}$ so that for $\omega \geq \omega_{0} \angle G(j \omega)<-\pi / 2$ rad. Consequently if the digital implementation of the control law (4) is modelled in continuous-time as the original controller connected in series with a time delay, the resulting system will be unstable.

\section{Example 1 (Digital implementation destroys stability)}

Consider a continuous-time system

$$
\left(p^{2}+5 p+1\right) y(t)=(p+1) u(t)
$$

where $p:=\frac{d}{d t}$ and $t \in[0, \infty)$. The system is supposed to track a reference signal $r(t)=\sin (t)$. The control is chosen to be

$$
u(t)=u(t-T)+e(t)
$$

The corresponding error signal $e(t)$ is shown in top part of Fig. 2 , which suggests asymptotic convergence. To make the simu-
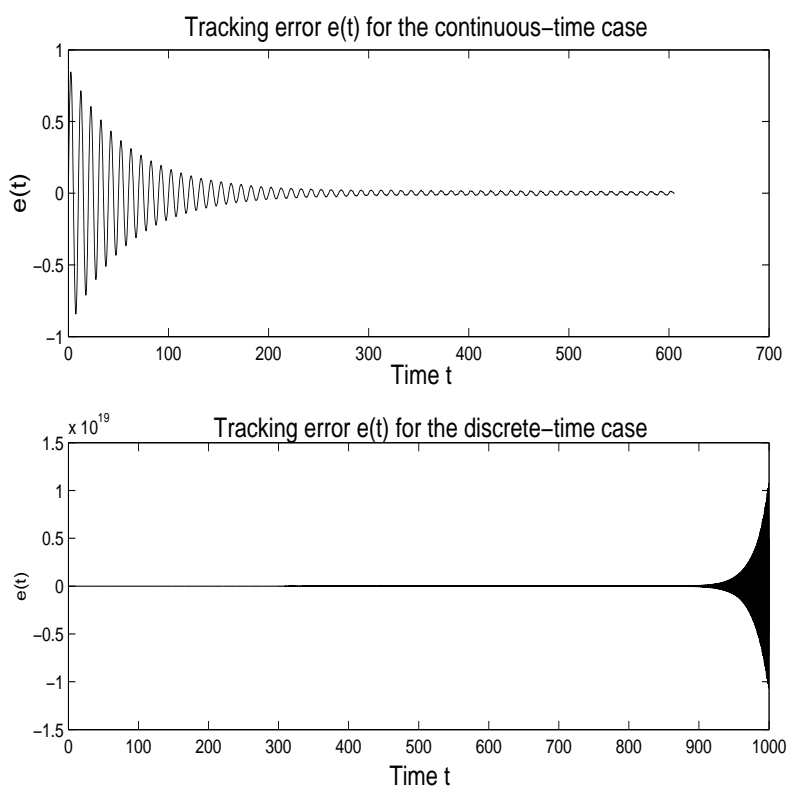

Figure 2: Tracking error $e(t)$ for Example 1

lation more realistic, the simulation model is modified into a hybrid simulation model in order to take into account the effect of sampling. This achieved by adding zero-order sampling for both $u(t)$ and $y(t)$, which models the effect of $A / D$ and to $D / A$ conversion. The sampling interval is chosen to be 0.1 seconds. Furthermore, the delay-line is implemented with a digital filter but the plant is simulated as if it was a continuous-time system. The resulting tracking error is shown for the hybrid simulation model in the bottom part of Fig. 2, which shows instability, just as the theory suggests.

\section{A new optimality based algorithm for discrete-time repetitive control}

In this section a new algorithm is introduced for discrete-time Repetitive Control. The algorithm design is done by combining the polynomial systems approach presented in (Blomberg and 
Ylinen, 1983) and optimal control. As a starting point consider a process model defined for $t \in \mathbb{Z}$

$$
A\left(z^{-1}\right) y(t)=B\left(z^{-1}\right) u(t)
$$

where $A\left(z^{-1}\right), B\left(z^{-1}\right) \in \mathbb{C}\left[z^{-1}\right]$ and $z^{-1}$ is the backward shift-operator, i.e. $\left(z^{-1} v\right)(k)=v(k-1)$ for $v(k) \in \mathbb{R}^{\mathbb{Z}}$. From now on it is assumed that process model (15) is both controllable and observable. Furthermore, a $T$-periodic reference signal $r(t)$ is given, i.e. $r(t+T)=r(t)$ and the control design objective is to make the output $y(t)$ to track this reference signal as accurately as possible by using a suitable feedback controller. As a starting point note that because the reference signal is $T$-periodic, the polynomial $D\left(z^{-1}\right)=1-z^{-T}$ is a annihilator (or an internal model) for $r(t)$, i.e.

$$
D\left(z^{-1}\right) r(t)=r(t)-z^{-T} r(t)=r(t)-r(t-T)=0 .
$$

By multiplying both sides of (15) with $D\left(z^{-1}\right)$ gives

$$
\begin{aligned}
& D\left(z^{-1}\right) A\left(z^{-1}\right) y(t)=D\left(z^{-1}\right) B\left(z^{-1}\right) u(t) \\
& =B\left(z^{-1}\right) D\left(z^{-1}\right) u(t) \\
& B\left(z^{-1}\right) \tilde{u}(t)
\end{aligned}
$$

where $\tilde{u}(t):=u(t)-u(t-T)$. Furthermore the left-hand side of (17) can be written as

$$
\begin{aligned}
& D\left(z^{-1}\right) A\left(z^{-1}\right) y(t)=A\left(z^{-1}\right) D\left(z^{-1}\right) y(t) \\
& =A\left(z^{-1}\right)(y(t)-y(t-T)) \\
& =A\left(z^{-1}\right)(y(t)-r(t)+r(t-T)-y(t-T)) \\
& =A\left(z^{-1}\right)(-e(t)+e(t-T)) \\
& =-D\left(z^{-1}\right) A\left(z^{-1}\right) e(t)=\tilde{A}\left(z^{-1}\right) e(t) .
\end{aligned}
$$

where $\tilde{A}\left(z^{-1}\right):=-D\left(z^{-1}\right) A\left(z^{-1}\right)$. Combining (17) and (18) gives

$$
\tilde{A}\left(z^{-1}\right) e(t)=B\left(z^{-1}\right) \tilde{u}(t)
$$

which is a controllable and observable dynamical system, if $D\left(z^{-1}\right)$ and $B\left(z^{-1}\right)$ do not have common factors. Consequently by using the internal model $D\left(z^{-1}\right)$ the original tracking problem is converted into a regulation problem, i.e the control objective is to find a feedback controller that drives the output $e(t)$ of the modified system (19) to zero. There in fact exist several different methods for achieving this. One of the more straightforward approaches is to use optimal control. As a starting point note that (19) has a state-space representation

$$
\begin{aligned}
& x_{m}(t+1)=A_{m} x_{m}(t)+B_{m} \tilde{u}(t) \\
& e(t)=C_{m} x_{m}(t)
\end{aligned}
$$

where the dimension of $x_{m}(\cdot)$ is $n+T$, and $n$ is the order of the original process model (15). Consider now the optimisation problem

$$
\min _{\tilde{u} \in l_{2}} J\left(\tilde{u}, x_{m}(0)\right)
$$

where

$$
\begin{aligned}
& J\left(\tilde{u}, x_{m}(0)\right)=\sum_{i=1}^{\infty} e(i)^{T} Q e(i)+\tilde{u}^{T}(i) R \tilde{u}(i) \\
& =\sum_{i=1}^{\infty} x_{m}(i)^{T} C_{m}^{T} Q C_{m} x_{m}(i)+\tilde{u}^{T}(i) R \tilde{u}(i)
\end{aligned}
$$

and $Q$ and $R$ are symmetric positive-definite weighting matrices. It is a well-known result from optimal control theory that the solution of the optimisation problem (21) is given by the control law $\tilde{u}(t)=-K x_{m}(t)$ or

$$
u(t)=u(t-T)-K x_{m}(t)
$$

where $K$ is given by the equation

$$
K=\left(B_{m}^{T} S B_{m}+R\right)^{-1} B_{m}^{T} S A_{m}
$$

and $S$ is obtained from the algebraic Riccati equation

$$
S=A_{m}^{T}\left[S-S B_{m}\left(B_{m}^{T} S B_{m}+R\right)^{-1} B_{m}^{T} S\right] A_{m}+Q
$$

Unfortunately, in practise it is impossible to measure the state $x_{m}(\cdot)$ directly. However, it is still possible to construct an observer for the state $x_{m}(\cdot)$, i.e. the states are estimated with the following equation

$$
\hat{x}_{m}(t+1)=A_{m} \hat{x}_{m}(t)+B_{m} \tilde{u}(t)+L\left(e(t)-C_{m} x_{m}(t)\right)
$$

where $L$ is the observer gain and the control law becomes

$$
u(t)=u(t-T)-K \hat{x}_{m}(t)
$$

Note that it is easy to take noise into account in the proposed algorithm: suppose that (20) would also have noise terms $w(t)$ and $v(t)$ in the following way,

$$
\begin{aligned}
& x_{m}(t+1)=A_{m} x_{m}(t)+B_{m} \tilde{u}(t)+G w(t) \\
& e(t)=C_{m} x_{m}(t)+v(n)
\end{aligned}
$$

where $w(t)$ and $v(t)$ are zero mean Gaussian noise. Conceptually $w(t)$ describes uncertainty in the state-space model, whereas $v(t)$ describes uncertainty in the measurement process. If the covariance matrix $Q_{n}$ of $v(t)$ and the covariance matrix $R_{n}$ of $w(t)$ are known, it is possible to find an optimal observer gain $L$ that minimises the variance of the estimation error. It is also a standard result in optimal control (see (Lewis and Syrmos, 1995)) that by combining the optimal feedback controller and optimal observer the resulting closed loop system is stable, and hence the expected value of $e(t)$ will go to zero as $t \rightarrow \infty$. The flow-diagram of the proposed algorithm is shown in Fig 3.

Remark 2 It is important to understand that this approach also works for more complex reference signals, the only requirement is that the reference signal has an annihilator polynomial, and that this polynomial does not have common factors with $B\left(z^{-1}\right)$-polynomial in the process model (15). A typical example would be a multi-periodic reference signal $r(t)=r_{1}(t)+r_{2}+\cdots+r_{n}(t)$ where $r_{i}(t)=r_{i}\left(t+T_{i}\right)$. In this case it is easy to show that the annihilator polynomial is

$$
D\left(z^{-1}\right)=D_{1}\left(z^{-1}\right) D_{2}\left(z^{-1}\right) \ldots D_{n}\left(z^{-1}\right)
$$

where $D_{i}\left(z^{-1}\right)=1-z^{-T_{i}}$. It is also straightforward to show that if there is a T-periodic load disturbance acting on the input signal, the same controller structure is able to learn the correct control action to cancel out the effect of this disturbance on the output signal $y(t)$. All the results in this section can be extended with some effort to include MIMO-systems, which will be reported separately. 


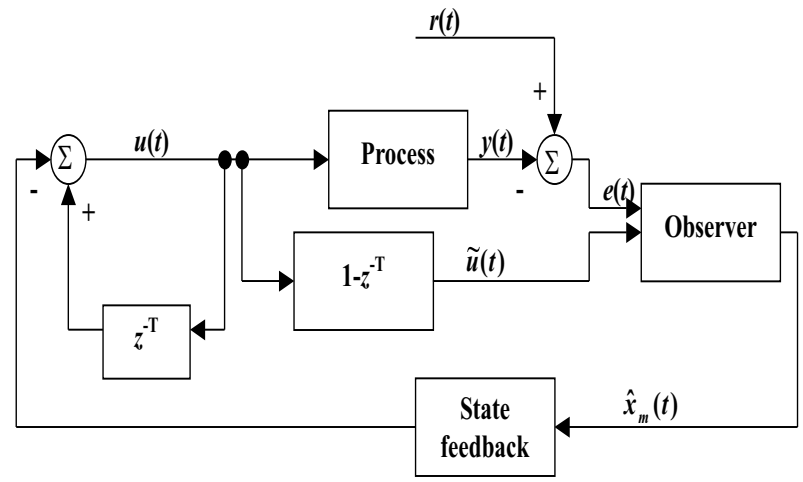

Figure 3: The flow diagram of the Repetitive Controller

\section{Simulation examples}

As a first simulation example consider the following following noise-free dynamical system:

$$
G\left(z^{-1}\right)=\frac{0.2011 z^{-1}-0.06241 z^{-1}}{1-0.1851 z^{-1}+0.006783 z^{-2}}
$$

The objective is to make this system to follow the repetitive reference signal in Fig. 4. The free parameters of the algorithm

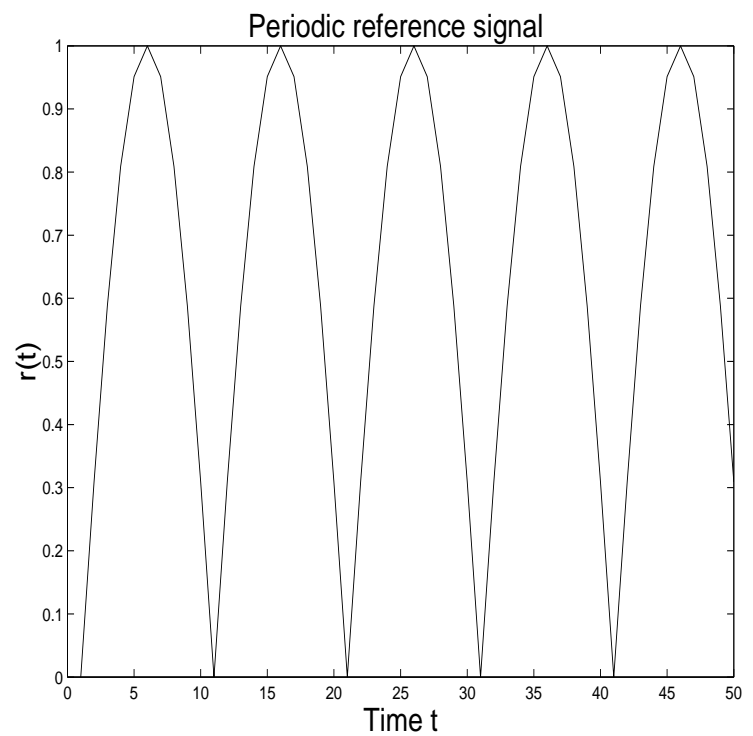

Figure 4: The periodic reference signal for the first simulation example

were selected in the following way: the weighting matrices are $Q=10, R=1$, and the observer gain $L$ was selected by using a Kalman-filtering approach. In the Kalam filter approach it was assumed that the covariance matrices $Q_{n}$ and $R_{n}$ are equality to identity matrices. Fig. 5 shows the $l_{2}$-norm of the tracking error for each period ('iteration'), and the tracking error goes to zero as the theory suggests.

As a second simulation example consider again the same dy-

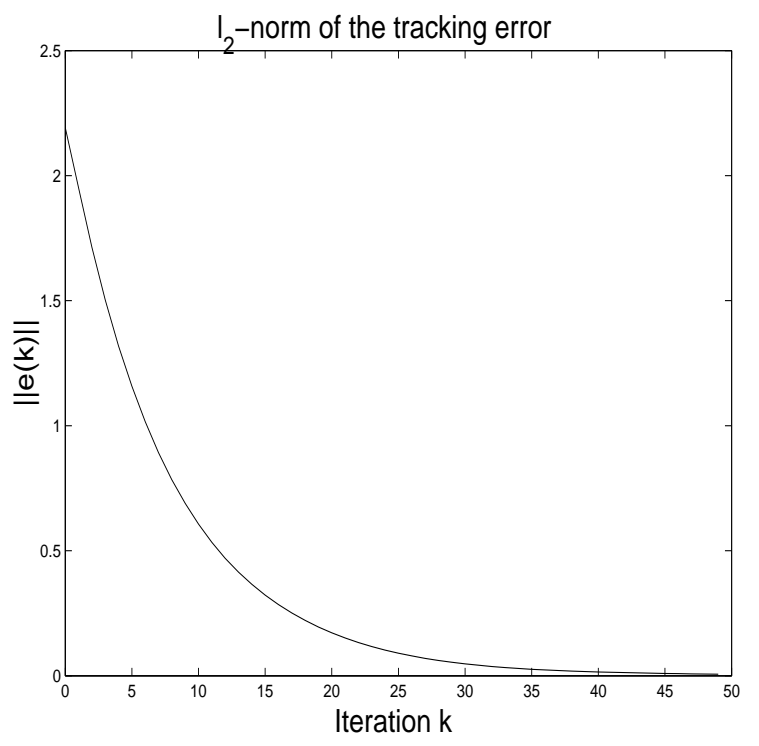

Figure 5: The norm of the tracking error for the first simulation example as a function of the period $k$

namical system (30). This time the system is supposed to track a multi-periodic reference signal $r(t)=\sin \left(2 \pi t / T_{1}\right)+$ $\sin \left(2 \pi t / T_{2}\right)$ where $T_{1}=11$ and $T_{2}=20$ (i.e. the lengths of the periods are not comeasurable). The internal model becomes

$$
\begin{aligned}
& D\left(z^{-1}\right)=\left(1-z^{-T_{1}}\right)\left(1-z^{-T_{2}}\right) \\
& =1-z^{-T_{1}}-z^{-T_{2}}+z^{-\left(T_{1}+T_{2}\right)}
\end{aligned}
$$

The algorithm was run with the same settings as in the previous example. Fig. 6 shows the the tracking error $e(t)$, which converges to zero.

Remark 3 Note that in each simulation the controller was initialised with a poor 'initial guess' $u(t)=0$ for $t \in[0, T]$. In practise, however, classical feedback control can be used to find quickly a reasonably accurate estimate of the input function $u(t)$ that gives perfect tracking, and when this estimate is available, the RC algorithm is switched on.

\section{Conclusions}

In this paper it was shown that the discretisation of a class of repetitive controllers results in instability. This is a major drawback, because according to the internal model principle, a repetitive controller has to contain a delay line, and in practise the delay line cannot be implemented with analogue components.

In order to overcome this problem, a new optimality based Repetitive Control algorithm was suggested in this paper. Furthermore, it was shown that under mild controllability/observability conditions the algorithm will result in asymptotic convergence.

As a future research it would worthwhile to take into account 


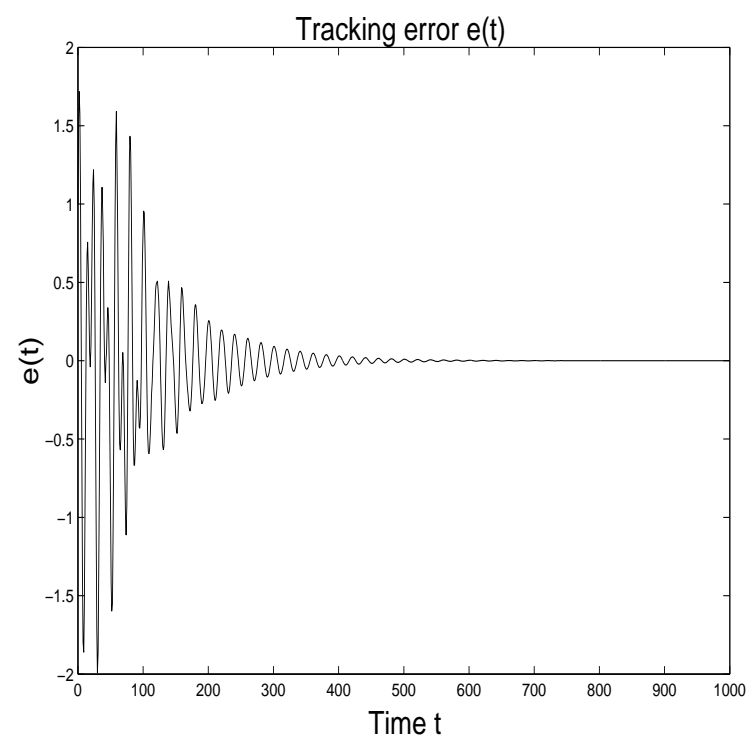

Figure 6: The tracking error $e(t)$ for the second simulation example

modelling uncertainty. However, this should be rather straightforward, because the design of the repetitive controller can be done by using standard techniques from $\mathrm{H}_{2}$ and $H_{\infty}$ theory. The sensitivity of the approach to noise in the input and output signals is another important topic for future research.

\section{Acknowledgements}

J. Hätönen was supported by the EU TMR contract No FMRXCT970137 (Nonlinear Control Network) and is now supported by the EPSRC contract No GR/R74239/10.

\section{References}

Anderson, B.D.O. and S. Vongpanithred (1973). Network analysis and synthesis: A modern system theory approach. Prentice Hall, Englewood Cliffs, NJ.

Arimoto, S. and T. Naniwa (2000). Equivalence relations between learnability, output-passivity and strict positive realness. International Journal of Control 73(10), 824-831.

Blomberg, H. and R. Ylinen (1983). Algebraic Theory for Multivariable Linear Systems. Academic Press.

Desoer, C.A. and M. Vidyasagar (1975). Feedback Systems:Input-Output Properties. Academic Press Inc.

Francis, B.A. and W.M. Wonhan (1975). The internal model principle for linear multivariable regulators. Appl. Math. Opt. pp. 107-194.

Fung, R.F., J.S Huang, C.G. Chien and Y.C. Wang (2000). Design and application of continuous time controller for ro- tation mechanisms. International journal of mechanical sciences 42, 1805-1819.

Garimella, S.S. and K. Srinivasan (1996). Application of repetitive control to eccentricity compensation in rolling. Journal of dynamic systems measurement and controlTransactions of the ASME 118, 657-664.

Inouye, T., M. Nakano, T. Kubo, S. Matsumoto and H. Baba (1981). High accuracy control of a proton synchrotron magnet power supply. In: Proceedings of the 8th IFAC World Congress.

Kaneko, K. and R. Horowitz (1997). Repetitive and adaptive control of robot manipulators with velocity estimation. IEEE Trans. on robotics and automation 13, 204-217.

Kobayashi, Y., T. Kimuara and S. Yanabe (1999). Robust speed control of ultrasonic motor based on $H_{\infty}$ control with repetitive compensator. JSME Int. Journal Series C 42, 884-890.

Lewis, F. L. and V. L. Syrmos (1995). Optimal Control. John Wiley \& Sons, Inc.

Ogata, K. (1973). Discrete-time Control Systems. Prentice Hall, Englewood Cliffs, NJ.

Owens, D.H., L.M. Li and S.P. Banks (2001). MIMOmultiperiodic repetitive control systems: a Liapunov analysis. In: Proceedings of the IFAC Worksop on periodic systems and control.

Yamamoto, Y. (1993). Essays on Control:Perspectives in the theory and its applications. Chap. Learning Control and Related Problems in Infinite-Dimensional Systems, pp. 191-222. Birkhauser.

Zhou, K., J.C. Doyle and K. Glover (1996). Robust Optimal Control. Prentice-Hall. 